\title{
ON QUALITATIVE PROPERTIES OF SOLUTIONS OF QUASILINEAR ELLIPTIC EQUATIONS WITH STRONG DEPENDENCE ON THE GRADIENT
}

\author{
JADRANKA KRALJEVIĆ \\ University of Zagreb, Croatia
}

\begin{abstract}
We are interested in the regularity of positive, spherically symmetric solutions of a class of quasilinear elliptic equations involving the $p$-Laplace operator, with an arbitrary positive growth rate $e_{0}$ on the gradient on the right-hand side. We study the regularity of a class of strong and weak solutions at the origin. Furthermore, we find some conditions under which strong solutions are classical.
\end{abstract}

\section{INTRODUCTION}

This work is a continuation of studies of spherically symmetric solutions of quasilinear elliptic equations in the ball, see [3-7]. Also, some results of this studies has been applied in [2]. While in these references the emphasis was put mostly on the problem of existence and nonexistence of solutions, here we concentrate on the problem of regularity of solutions near the origin. The main results are contained in Theorem 1.3 and Corollaries 1.5 and 1.7. The proofs make use of a suitable integral representation of solutions (see [6]), introduced in [5], and further extended in [3].

This paper provides some generalizations of the main results in [7], where the problem of existence of positive symmetric strong solutions of quasilinear elliptic equations has been studied, involving $p$-Laplacian in the ball. These equations allowed simultaneous strong dependence of the right-hand on both the unknown function and its gradient and also studied a posteriori regularity of solutions. Here, we consider some qualitative properties of a class of strong

2010 Mathematics Subject Classification. 35C15, 35J60, 45 J05.

Key words and phrases. Quasilinear elliptic, positive strong solution, weak solution, regularity, classical solution. 
and weak solutions of quasilinear elliptic equations with strong dependence on the gradient such that the exponent on the gradient is any positive number.

In this paper we study the regularity of positive, spherically symmetric solutions of the following quasilinear elliptic problem:

$$
\left\{\begin{aligned}
-\Delta_{p} u & =\tilde{g}_{0}|x|^{m}+\tilde{f}_{0}|\nabla u|^{e_{0}} \quad \text { in } B \backslash\{0\}, \\
u & =0 \text { on } \partial B, \\
u(x) & \text { spherically symmetric and decreasing. }
\end{aligned}\right.
$$

Here $B$ is an open ball of radius $R$ centered at the origin in $\mathbb{R}^{N}, 1<p<$ $\infty$, and $\Delta_{p} u=\operatorname{div}\left(|\nabla u|^{p-2} \nabla u\right)$ is the $p$-Laplacian. The Lebesgue measure (volume) of $B$ in $\mathbb{R}^{N}$ is denoted by $|B|$, and the volume of the unit ball is denoted by $C_{N}$. The dual exponent of $p>1$ is defined by $p^{\prime}=\frac{p}{p-1}$. We assume that $\tilde{g}_{0}, \tilde{f}_{0}$ and $e_{0}$ are positive real numbers. By a strong solution we mean a function $u \in C^{2}(B \backslash\{0\}) \cap C(\bar{B})$ which satisfies (1.1) pointwise.

In [4] we showed that the exponent $e_{0}=p-1$ on the gradient in (1.1) is critical in the following sense: if $0<e_{0}<p-1$ problem (1.1) is solvable for all positive $\tilde{g}_{0}$ and $\tilde{f}_{0}$ (which is known result), while for $e_{0}>p-1$ we have nontrivial existence and nonexistence regions in the positive quadrant of $\left(\tilde{g}_{0}, \tilde{f}_{0}\right)$-plane. The main results in [4] have been given in the next two theorems.

Theorem 1.1. (see [4]). Assume that $m>\max \{-p,-N\}, N \geq 2$.

(a) If $0<e_{0}<p-1$ and $m \leq e_{0} /\left(p-e_{0}-1\right)$, then the problem (1.1) possesses a strong solution for all positive $\tilde{g}_{0}$ and $\tilde{f}_{0}$.

(b) Let $e_{0}>p-1$. Then there exist two explicit positive constants $\tilde{C}_{1}$ and $\tilde{C}_{2}, \tilde{C}_{1}<\tilde{C}_{2}$, such that

(b1) if $m \leq e_{0} /\left(p-e_{0}-1\right), m \neq-1$ and if

$$
\tilde{f}_{0} \tilde{g}_{0}^{\frac{e_{0}}{p-1}-1} \leq \tilde{C}_{1}
$$

then there exists a strong solution of quasilinear elliptic problem (1.1);

(b2) if $m<e_{0} /\left(p-1-e_{0}\right)$ and

$$
\tilde{f}_{0} \tilde{g}_{0}^{\frac{e_{0}}{p-1}-1} \geq \tilde{C}_{2}
$$

then problem (1.1) has no strong solutions.

The explicit values of $\tilde{C}_{1}$ and $\tilde{C}_{2}$ can be expressed in dependence on $N$, $p, m, T$ and $e_{0}$ (see $\left.[3,4]\right)$.

We have proved the existence result stated in Theorem 1.1 by studying the corresponding singular ODE, (see $[3,4])$ :

$$
\frac{d \omega}{d t}=g_{0} \gamma t^{\gamma-1}+f_{0} \frac{\omega(t)^{\delta}}{t^{\varepsilon}} \quad, \quad t \in(0, T)
$$


where $\gamma, \delta, \varepsilon, g_{0}, f_{0}$, are positive constants, depending on $N, p, m, \tilde{f}_{0}, \tilde{g}_{0}$ and $e_{0}$.

For $\varepsilon>0$ this equation is singular at $t=0$. An existence result of ODE (1.4) was obtained for $\omega$ in the set:

$$
D_{M}=\left\{\varphi \in C([0, T]): 0 \leq \varphi(t) \leq M t^{\gamma}\right\},
$$

for $\gamma>0$ and for suitably chosen constant $M$, which enables to apply Schauder's fixed point theorem.

Following the terminology introduced in [3], we say that $u$ is an $\omega$-solution of a quasilinear elliptic problem (1.1), if it is a strong solution which can be obtained as an integral representation

$$
u(x)=\int_{C_{N}|x|^{N}}^{|B|} \frac{\omega(t)^{p^{\prime}-1}}{t^{p^{\prime}\left(1-\frac{1}{N}\right)}} d t,
$$

generated by a solution $\omega$ of equation (1.4), with additional requirement that $0 \leq \omega(t) \leq M t^{\gamma}$, for suitably chosen constant $M>0$. Furthermore, the following relation holds for all $r \in(0, R]$ :

$$
\tilde{u}^{\prime}(r)=-|\nabla u|=-N C_{N}^{1 / N}\left(\frac{\omega(s)}{s^{1-\frac{1}{N}}}\right)^{p^{\prime}-1}, \quad s=C_{N}|x|^{N},
$$

where $\tilde{u}(r)$ is defined by $\tilde{u}(r)=u(x), r=|x|$. Under some additional conditions, strong solutions of (1.1) described above are also weak solutions in the Sobolev space $W^{1, p}(B)$.

Theorem 1.2 ([4]). Assume that $N \geq 2, m>\max \{-p,-N\}$, and $m>$ $-1-\frac{N(p-1)}{e_{0}}$. Then any $\omega$-solution of quasilinear elliptic problem (1.1) is also a weak solution.

\section{MAin Results}

In Theorem 1.1 we have proved more than just existence of strong solutions: there exist $\omega$-solutions of quasilinear elliptic problem (1.1). It is easy to see that $\omega$-solutions are in $C^{\infty}(\bar{B} \backslash\{0\})$. Indeed, from (1.4) for any solution $\omega \in D$ of (1.4) we can see inductively that $\omega \in C^{k}((0, T))$ for all $k \in \mathbb{N}$. Using integral representation (1.6) for any strong $\omega$-solution $u$ we see to be in $C^{k}(\bar{B} \backslash\{0\})$ for all $k \in \mathbb{N}$, hence it is in $C^{\infty}(\bar{B} \backslash\{0\})$. The main result of this section is Theorem 1.3 about the regularity of $\omega$-solutions of (1.1) at the origin. This result extends [7, Theorem 3.2]. Namely, in [7], the author in discussion of regularity of solutions at the origin considered equations with the natural growth on the gradient, that is, when the exponent on the gradient is $e_{0}=p$. Here, we allow that the exponent on the gradient $e_{0}$ could be any positive number. Also in Theorem 1.3, and in Lemma 1.9, we give some results about the higher order derivative of the solutions at the origin. As a special case of this generalisation, for the second order derivative, with some 
other assumptions given in Lemma 1.6, we find the conditions under which the strong solution is classical.

THEOREM 1.3 (Regularity of $\omega$-solutions at the origin). Let $m>$ $\max \{-p,-N\}, N \geq 2, m \leq \frac{e_{0}}{p-e_{0}-1}$ and $k \in \mathbb{N}$. Assume that for a given $k \geq 2$, we have $m \notin\{0, N, 2 N, \ldots,(k-2) N\}$. Let $u$ be any $\omega$ solution of quasilinear elliptic problem (1.1) and let $\tilde{u}$ be the function given by $\tilde{u}(r)=u(x)$, where $r=|x|$. Let $d_{k}$ be a real constant depends on $m, p$ and $N$ defined in Lemma 1.9 below.

(a) If $m<k(p-1)-p$ and $d_{k} \neq 0$, then $\lim _{r \rightarrow 0} \tilde{u}^{(k)}(r)=\left(\operatorname{sign} d_{k}\right) \cdot \infty$

(b) If $m \geq k(p-1)-p$, then

$$
\lim _{r \rightarrow 0} \tilde{u}^{(k)}(r)= \begin{cases}d_{k} \tilde{g}_{0}^{p^{\prime}-1} & \text { if } m=k(p-1)-p, \\ 0 & \text { if } m>k(p-1)-p .\end{cases}
$$

We shall give the proof of this theorem in the next section.

REMARK 1.4. We see that if $m$ increases, then the regularity of $\omega$-solution $u$ at $x=0$ also increases. Let us mention that (see Lemma 1.9 below):

$$
d_{1}=-\frac{1}{(m+N)^{p^{\prime}-1}}<0 \quad \text { and } \quad d_{2}=-\frac{m+1}{(p-1)(m+N)^{p^{\prime}-1}} .
$$

It follows that for $m>-1$ we have $d_{2}<0$, while for $m<-1$ we have $d_{2}>0$. We can consider this case because $N \geq 2$ and $m>\max \{-p,-N\}$. We have the following conclusion about the qualitative properties of $\omega$-solution of quasilinear elliptic problem (1.1):

(a) If $m<-1$, then there exists $\tilde{\varepsilon}>0$, such that $\tilde{u}$ is strictly convex for $0<r<\tilde{\varepsilon}$, since then $\tilde{u}^{\prime \prime}(r)>0$ on $(0, \tilde{\varepsilon})$.

(b) If $m>-1$, then there exists $\tilde{\varepsilon}>0$, such that $\tilde{u}$ is strictly concave for $0<r<\tilde{\varepsilon}$, since then $\tilde{u}^{\prime \prime}(r)<0$ on $(0, \tilde{\varepsilon})$.

In the case $k=1$ we obtain from Theorem 1.3 a result on the sharpness of the spike of the graph of the solution $u$ of (1.1) at the origin in dependence on the $m$. It is interesting that the sharpness of the spike does not depend of the coefficient $\tilde{f}_{0}$ near the gradient.

Corollary 1.5. Let $m>\max \{-p,-N\}, N \geq 2$.

(a) Assume that $0<e_{0}<p-1$ and $m \leq \frac{e_{0}}{p-e_{0}-1}$. Let $u$ be any $\omega$-solution of (1.1) and $\tilde{u}(r)=u(x)$, for $r=|x|$. Then:

$$
\lim _{r \rightarrow 0} \tilde{u}^{\prime}(r)= \begin{cases}-\infty, & \text { for } m<-1, \\ -\left(\frac{\tilde{g}_{0}}{-1+N}\right)^{p^{\prime}-1}, & \text { for } m=-1 \\ 0 & \text { for } m>-1\end{cases}
$$


(b) Assume that $e_{0}>p-1, m \leq \frac{e_{0}}{p-e_{0}-1}$, and $m \neq-1$. Let $u$ be any $\omega$-solution of (1.1) and $\tilde{u}(r)$, defined below. Then:

$$
\lim _{r \rightarrow 0} \tilde{u}^{\prime}(r)= \begin{cases}-\infty, & \text { for } m<-1, \\ 0 & \text { for } m>-1 .\end{cases}
$$

Proof. The proof follows directly from Theorem 1.3, using (1.1). For $m=-1$ in the case for $0<e_{0}<p-1$, we have

$$
\lim _{r \rightarrow 0} \tilde{u}^{\prime}(r)=-\left(\frac{\tilde{g}_{0}}{-1+N}\right)^{p^{\prime}-1} .
$$

For $k=2$ we obtain sufficient conditions for existence of a classical solution of (1.1), which is a special case of Theorem 1.3. Let us recall that $u$ is a classical solution of (1.1) if $u \in C^{2}(\bar{B})$ and if $u$ satisfies (1.1) pointwise.

We shall need the following lemma.

Lemma 1.6. Let $u \in C^{2}(B \backslash\{0\}) \cap C(\bar{B})$ and let $\tilde{u}(r)=u(x)$, for $r=|x|$. If $\lim _{r \rightarrow 0} \tilde{u}^{\prime \prime}(r)=0$ and if $\left|\tilde{u}^{\prime}(r)\right| \leq C r^{\alpha}$, where $\alpha>1$, and $C$ is some positive constant, then $u \in C^{2}(\bar{B})$.

Corollary 1.7. Let $k=2, m \neq 0$ and let $m>\max \{-p,-N\}, N \geq 2$, $m \leq \frac{e_{0}}{p-e_{0}-1}$. Let $u$ be any $\omega$-solution of (1.1) and $\tilde{u}(r)=u(x)$, for $r=|x|$. Then:

$$
\lim _{r \rightarrow 0} \tilde{u}^{\prime \prime}(r)= \begin{cases}-\infty, & \text { for }-1<m<p-2, \\ -\frac{m+1}{p-1} \cdot\left(\frac{\tilde{g}_{0}}{m+N}\right)^{p^{\prime}-1}, & \text { for } m=p-2, \\ 0 & \text { for } m>p-2 .\end{cases}
$$

In particular for $m>p-2$ any $\omega$-solution of (1.1) is also a classical solution.

Proof. The proof follows directly from Theorem 1.3, using (1.1) and (1.2). For the $\omega$-solution $u$ to be also the classical solution, we have to prove that all partial derivatives of the second order are continuous at $x=0$. This follows directly from Lemma 1.6 and Theorem 1.3, from which it follows that for $m>p-2$, we have $\tilde{u}^{\prime \prime}(r) \rightarrow 0$ when $r \rightarrow 0$, and

$$
\frac{1}{r} \tilde{u}(r) \sim \frac{1}{r} \tilde{K} r^{\frac{p}{p^{\prime}}(m+1)}=\tilde{K} r^{\frac{m+1}{p-1}-1} \rightarrow 0
$$

where $\tilde{K}$ is some positive constant, if and only if $\frac{m+1}{p-1}-1>0$, that is, for $m>p-2$.

\section{Proofs of Theorem 1.3 And of auxiliary Results}

Let us first introduce some notation. For $\gamma \in \mathbb{R}$ and $n \in \mathbb{N}$ we define $(\gamma)_{n}=\gamma(\gamma-1)(\gamma-2) \ldots(\gamma-n+1)$ and $(\gamma)_{0}=1$. For any two real functions $a, b:(0, T] \rightarrow \mathbb{R}$, we write $a(t) \sim b(t)$ when $t \rightarrow 0$, if $\lim _{t \rightarrow 0} \frac{a(t)}{b(t)}=1$.

The next two lemmas will be used in the proof of Theorem 1.3. 
LEMMA 1.8. Assume that $\delta>0, \delta>\frac{\varepsilon-1}{\gamma}+1, \gamma>0, N \geq 2$. Let $\omega$, $0 \leq \omega(t) \leq M t^{\gamma}$ be a solution of (1.4). Assume that $n \in \mathbb{N} \cup\{0\}$ and for $n \geq 3$ we assume that $\gamma \notin\{1,2, \ldots, n-1\}$. Then

$$
\omega^{(n)}(t)=(\gamma)_{n} t^{\gamma-n} g_{0}+O\left(t^{\gamma \delta-\varepsilon+1-n}\right), \quad \text { when } t \rightarrow 0 .
$$

In particular:

$$
\omega^{(n)}(t) \sim(\gamma)_{n} t^{\gamma-n} g_{0}, \quad \text { when } t \rightarrow 0 .
$$

Lemma 1.9. Let $m>\max \{-p,-N\}, N \geq 2, m \leq \frac{e_{0}}{p-e_{0}-1}$ and $k \in \mathbb{N}$. If $k \geq 2$, we assume that $m \notin\{0, N, 2 N, \cdots(k-2) N\}$. Then for any $\omega$-solution $u$ of quasilinear elliptic problem (1.1) we have

$$
\lim _{r \rightarrow 0} \frac{\tilde{u}^{(k)}(r)}{r^{\frac{p^{\prime}}{p}(m+1)-k+1}}=d_{k} \tilde{g}_{0}^{p^{\prime}-1},
$$

where $d_{k}=d_{k}(m, N, p)$ is a real constant, depending on $k, m, p$ and $N$ and the function $\tilde{u}:(0, R] \rightarrow \mathbb{R}$ is given by $\tilde{u}(r)=u(x), r=|x|, r \in(0, R]$.

The proofs of the results of Lemmas 1.8 and 1.9 easily follow by induction, so we omit it. In the proof of Lemma 1.9 we can obtain the explicit values of the real constant $d_{k}$ depending on $k, m, p$ and $N$. For some conclusion about the qualitative properties of $\omega$-solutions of quasilinear elliptic problem (1.1), we have the values of $d_{1}$ and $d_{2}$ :

$$
d_{1}=-\frac{1}{(m+N)^{p^{\prime}-1}}<0 \quad \text { and } \quad d_{2}=-\frac{m+1}{(p-1)(m+N)^{p^{\prime}-1}} .
$$

Now we prove Theorem 1.3.

Proof of Theorem 1.3. The claim follows directly from Lemma 1.9. Namely, from $m<k(p-1)-p$ it follows that $\frac{p^{\prime}}{p}(m+1)-(k-1)=\frac{m+1}{p-1}-k+1<$ 0 . Then from (1.3) and $d_{k} \neq 0$ we have that $\lim _{r \rightarrow 0} \tilde{u}^{(k)}(r)=\left(\operatorname{sign} d_{k}\right) \cdot \infty$. If $m=k(p-1)-p$ (which is equivalent with $\left.\frac{p^{\prime}}{p}(m+1)-k+1=0\right)$, then $\lim _{r \rightarrow 0} \tilde{u}^{(k)}(r)=d_{k} \tilde{g}_{0}^{p^{\prime}-1}$. For $m>k(p-1)-p$ we have $\lim _{r \rightarrow 0} \tilde{u}^{(k)}(r)=0$.

Now we prove Lemma 1.6.

Proof of Lemma 1.6. It suffices to see that all partial derivatives of the second order of the function $u$ are continuous at $x=0$. Since $r=|x|=$ $\sqrt{x_{1}^{2}+x_{2}^{2}+\cdots+x_{N}^{2}}$, we have

$$
\frac{\partial^{2} u}{\partial x_{i}^{2}}=\frac{\partial}{\partial x_{i}}\left(\tilde{u}^{\prime}(r) \frac{x_{i}}{r}\right)=\tilde{u}^{\prime \prime}(r) \frac{x_{i}^{2}}{r^{2}}+\tilde{u}^{\prime}(r) \frac{r^{2}-x_{i}^{2}}{r^{3}} .
$$


From $\frac{x_{i}^{2}}{r^{2}}=\frac{x_{i}^{2}}{x_{1}^{2}+\cdots+x_{N}^{2}} \leq 1$, it follows that

$$
\tilde{u}^{\prime \prime}(r) \frac{x_{i}^{2}}{r^{2}} \rightarrow 0, \quad \text { when } r \rightarrow 0 .
$$

Also,

$$
0 \leq \frac{r^{2}-x_{i}^{2}}{r^{3}} \leq \frac{r^{2}}{r^{3}}=\frac{1}{r} .
$$

We conclude that $\left|\tilde{u}^{\prime}(r)\right| \leq C r^{\alpha}$, where $\alpha>1$ and $C$ is some positive constant. We see that $\frac{1}{r}\left|\tilde{u}^{\prime}(r)\right| \leq \frac{1}{r} C r^{\alpha}=C r^{\alpha-1}$. Since $\alpha>1$, we have

$$
\tilde{u}^{\prime}(r) \frac{r^{2}-x_{i}^{2}}{r^{3}} \rightarrow 0, \quad \text { when } r \rightarrow 0 .
$$

For any $i, j=1, \ldots, N$ we obtain the same conclusion for

$$
\frac{\partial^{2} u}{\partial x_{i} \partial x_{j}}=\tilde{u}^{\prime \prime}(r) \frac{x_{j} x_{i}}{r^{2}}-\tilde{u}^{\prime}(r) \frac{x_{i} x_{j}}{r^{3}} .
$$

Since $\left|\frac{x_{i} x_{j}}{r^{2}}\right| \leq 1$, we have

$$
\tilde{u}^{\prime \prime}(r) \frac{x_{j} x_{i}}{r^{2}}-\tilde{u}^{\prime}(r) \frac{x_{i} x_{j}}{r^{3}} \rightarrow 0, \quad \text { when } r \rightarrow 0 .
$$

\section{ACKNOWLEDGEMENTS.}

The author is grateful to anonymous referees for their suggestions.

\section{REFERENCES}

[1] D. Gilbarg and N. S. Trudinger, Elliptic partial differential equations of second order, Springer-Verlag, Berlin, 1983.

[2] L. Horvat, J. Kraljević, D. Žubrinić and V. Županović, Positive solutions of polyharmonic equations with strong dependence on the gradient, Complex Var. Elliptic Equ. 52 (2007), 693-707.

[3] L. Korkut, M. Pašić and D. Žubrinić, A singular ODE related to quasilinear elliptic equations, Electron. J. Differential Equations 2000, No. 12, 37 pp.

[4] J. Kraljević, and D. Žubrinić, Quasilinear elliptic equations with positive exponent on the gradient, Glas. Mat. Ser. III 48(68) (2013), 391-402.

[5] M. Pašić, Nonexistence of spherically symmetric solutions for $p$-Laplacian in the ball, C. R. Math. Acad. Sci. Soc. R. Can. 21 (1999), 16-22.

[6] D. Žubrinić, Positive solutions of quasilinear elliptic systems with the natural growth in the gradient, Rend. Istit. Mat. Univ. Trieste 32 (2000), 65-102.

[7] D. Žubrinić, Solvability of quasilinear elliptic equations with strong dependence on the gradient, Abstr. Appl. Anal. 5 (2000), 159-173.

J. Kraljević

Department of Mathematics, Faculty of Economics

University of Zagreb

Kennedyev trg 6, 10000 Zagreb

Croatia

E-mail: jkraljevic@efzg.hr

Received: 11.10.2013. 